\title{
Intelligent Design of Brain-penetrant Compounds Based on Transporter- Conscious Drug Design
}

\section{Toshihiko Tashima}

Tashima Laboratories of Arts and Sciences, 1239-5 Toriyama-cho, Kohoku-ku, Yokohama, Kanagawa 222-0035, Japan

\section{Abstract}

In drug discovery and development, central nervous system (CNS) drugs should be delivered into brain. Nonetheless, drug permeability into brain may be blocked by the blood-brain barrier (BBB) due to the imperviousness of the tight junction between endothelial cells in CNS and excretion by MDR1 (P-gp) expressing in the apical (luminal) membrane of endothelial cells. However, SLC transporter-mediated transport at the $\mathrm{BBB}$ can solve this permeable problem. It is well-known that compounds possessing $\mathrm{N}$-containing groups are transported into brain across the BBB. This transportation was suggested to be associated with amine transporters. Thus, transporter-consciously designed drugs which possess $\mathrm{N}$-containing groups as transporter recognition unit can be effectively delivered into brain across the BBB. In this paper, possibility of CNS drugs based on transporter-conscious drug design is described.

\section{Publication History:}

Received: July 22, 2016

Accepted: September 21, 2016

Published: September 23, 2016

\section{Keywords:}

Transporter-conscious drug design, Drug design, Brain-penetrant compound, Drug delivery, BBB, ADMET

\section{Introduction}

In drug discovery and development, permeability of drugs across the cell membrane is generally a serious problem to overcome. Particularly, CNS drugs must penetrate the BBB into brain. So far several methods such as cell-penetrating peptide(CPP) transport systems using CPP-cargo conjugates or receptor-mediated transport (RMT) systems using the molecular Trojan horse (MTH) have been reported [1,2]. However, transporters [3] can solve these problems. Transporter proteins, which are tissue-specifically expressed, absorb or excrete materials such as nutrients and metabolites across the cell membrane and recognize the structures of their substrates through the transportation. The BBB is essentially attributed to the imperviousness of the tight junction between endothelial cells in CNS and excretion by MDR1 (P-gp) expressing in the apical (luminal) membrane of endothelial cells. MDR 1at the BBB excludes compounds just passing through the cell membrane by passive diffusion [4]. Thus, the tactic utilizing co-expressed SLC transporters in the apical (luminal) membrane of endothelial cells is one of alternative methods to deliver compounds such as drugs and imaging agents into brain across the BBB (Figure 1). Therefore, transporter-conscious drug design is very important [5]. In this short review, I will introduce the effective method of brain-penetrant drug design based on transporterconscious drug design.

\section{Discussion}

\section{Characterization of transporters}

Transporters are tissue-specifically expressed membrane proteins which absorb or excrete materials such as nutrients and metabolites by recognizing their structures to maintain physiological homeostasis totally in vivo and are divided into two families: $\mathrm{ABC}$ transporter super family and SLC transporter super family. SLC transporters [6] are secondary active influx proteins which absorb nutrients or endogenous compounds using the gradient energy of the transported materials. On the other hand, $\mathrm{ABC}$ transporters are primary active efflux proteins excreting many types of compounds as xenobiotics from cells using the chemical energy from ATP hydrolysis. MDR1 belongs to $\mathrm{ABC}$ transporter and are expressed in apical (luminal) membrane of capillary endothelial cells, small intestinal epithelial cells, and for some situations in cancer cells. Some types of drugs are excreted by MDR1 from the cells. Thus, drug delivery across the cell membrane using co-expressed SLC transporters near MDR1s is an excellent method to solve the membrane permeability problem. Transporter proteins recognize structurally their own substrates. Therefore, transporter-conscious drug design is a promising approach in drug discovery and development.

\section{Transporter-consciously designed drugs across the BBB}

It is well-known that compounds possessing $N$-containing groups penetrate the cell membrane across the BBB [5]. This fact suggests that these $\mathrm{N}$-containing compounds are transported by amine transporters. In fact, memantine, used for Alzheimer's disease, is concentrationdependently transported by some types of transporters, not by passive diffusion because amines form ammonium ions under physiological $\mathrm{pH}$ condition [7]. The candidate of amine transporters is suggested to be the proton-coupled organic cation $\left(\mathrm{H}^{+} / \mathrm{OC}\right)$ antiporter. However, the characterization of this $\mathrm{H}^{+} / \mathrm{OC}$ antiporter such as amino acid sequence and topology is unclear. Substrates of mouse $\mathrm{H}^{+} / \mathrm{OC}$ antiporter in apical membrane of capillary endothelial cells at the BBB by in in situ mouse brain perfusion assay are clonidine, quinidine, $p$-chloroamphetamine, cocaine, codeine, diacetylmorphine, diphenhydramine, MDMA, methadone, morphine, nalbuphine, nicotine, oxycodone, and tramadol [8]. Substrates of human $\mathrm{H}^{+} / \mathrm{OC}$ antiporter in apical membrane of capillary endothelial cells sat the $\mathrm{BBB}$ in in vitro assay using human CMEC/D3 cells are pyrilamine, oxycodone, quinidine, amantadine, and diphenhydramine [9]. Thus, compounds possessing $\mathrm{N}$-containing groups as transporter recognition unit can be substrates of this $\mathrm{H}^{+} / \mathrm{OC}$ antiporter, which was not competitively inhibited by TEA (OCT1-3, OCTN1, and OCTN2 substrate) or serotonin (PMAT substrate). LAT1, GluT1, SVCT2, and CNT2 in addition to this $\mathrm{H}^{+} / \mathrm{OC}$ antiporter are expressed in apical (luminal) membrane of capillary endothelial cells at the BBB.

"Corresponding Author: Dr. Toshihiko Tashima, Tashima Laboratories of Arts and Sciences, 1239-5 Toriyama-cho, Kohoku-ku, Yokohama, Kanagawa 2220035, Japan; E-mail: tashima_lab@yahoo.co.jp

Citation: Tashima T (2016) Intelligent Design of Brain-penetrant Compounds Based on Transporter-Conscious Drug Design. Int J Clin Pharmacol Pharmacother 1: 118. doi: http://dx.doi.org/10.15344/2016/ijccp/118

Copyright: (c) 2016 Tashima et al. This is an open-access article distributed under the terms of the Creative Commons Attribution License, which permits unrestricted use, distribution, and reproduction in any medium, provided the original author and source are credited. 


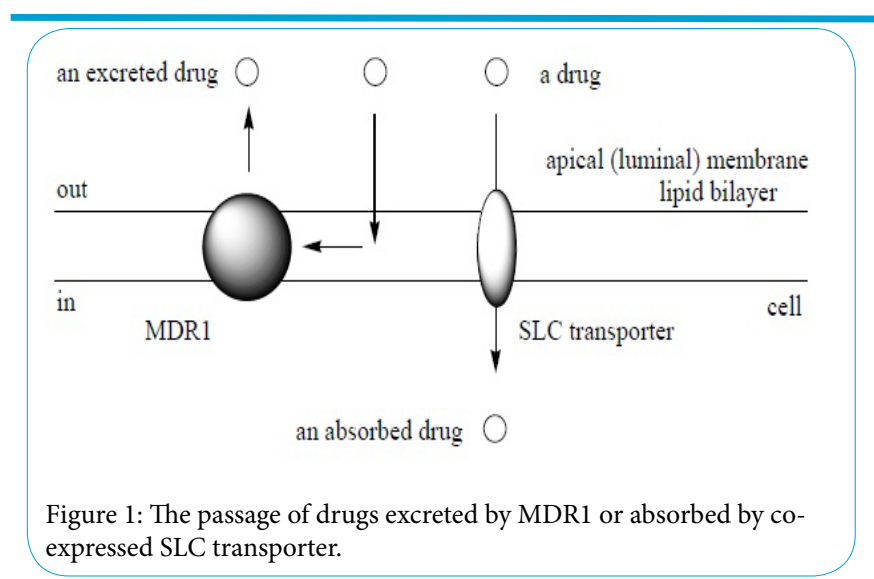

However, drug designs conducted based on such SLC transporters often utilize prodrug system in which parent drugs are conjugated with transporter recognition unit by a linker using the ester bond. The orally administered prodrug connected by the ester bond may be hydrolyzed in liver or blood serum before reaching the brain. Eventually, compounds possessing $N$-containing groups within a molecule based on transporter-conscious drug design might be superior to such prodrugs. This is one of the simplest methods because compactly designed compounds possessing $\mathrm{N}$-containing groups do not have accessory units such as transporter recognition units tethered to the parent drugs through the linker by cleavable bonds.

\section{Recently reported cases of brain-penetrant compounds}

Some brain-penetrant compounds possessing $\mathrm{N}$-containing groups have been reported. Recently reported brain-penetrant compounds are shown in Figure 2 and Table 1. Lu AF33241 (1) is a dual inhibitor of phosphodiesterase (PDE) 2A and PDE10A [10]. Compound (2) is a kappa opioid receptor (KOR) agonist [11]. Compound (3) is a 5-HT4 partial agonist [12]. Compound (4) is a kinesin spindle protein (KSP) inhibitor [13]. Compound (5) is a dual inhibitor of leucine zipper kinase (DLK, MAP3K12) [14]. Moreover, ${ }^{18} \mathrm{~F}-\mathrm{THK}-5105$ (6), ${ }^{18} \mathrm{~F}-\mathrm{THK}-5117$ (7), and ${ }^{18} \mathrm{~F}-\mathrm{THK}-523$ (8) are tau positron emission tomography (PET) imaging agents for Alzheimer's disease [15]. These brain-penetrant compounds share common structural features possessing $\mathrm{N}$-containing groups. These results suggested that $N$-containing groups functioned as transporter recognition unit towards transporters expressing at the BBB. Interestingly, it was likely that compounds with the dimethylamino group were transported greater than those with the methylamino group or the amino group. These findings will develop the method of transporter-conscious drug design.

\section{Brain-penetrant compounds possessing $\mathrm{N}$-containing groups as a prodrug}

The prodrugs possessing $\mathrm{N}$-containing groups as transient transporter recognition unit tethered to the parent drug by cleavable bonds can be delivered into brain by some types of amine transporters. Chlorambucil (CHL) was not transported into brain across the BBB. However, the prodrug of CHL, chlorambucil-scopine (CHLS) (Figure 3 ), was transported into brain across the BBB in energy-dependent manner both in in vitro assay using bEnd. 3 cells which were murine brain endothelial cells and in in situ rat brain perfusion assay. Not CHL but CHLS possessed the tertiary alkyl amino group. Thus, this transportation of CHLS was suggested to be based on amine transporters and actually was not inhibited by TEA (OCT1-3, OCTN1,

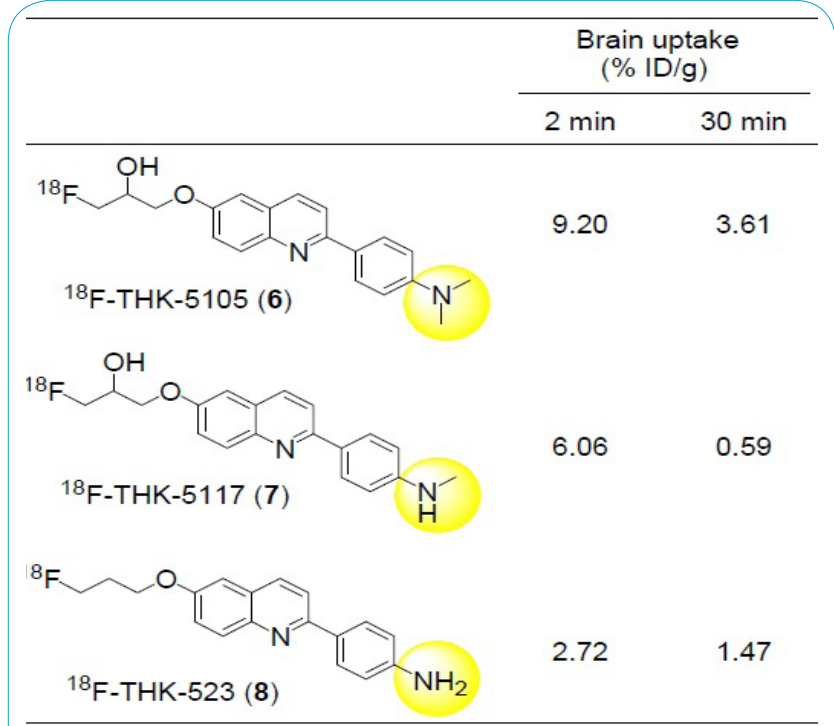

Table 1: The structures and the brain uptake of tau positron emission tomography (PET) imaging agents.

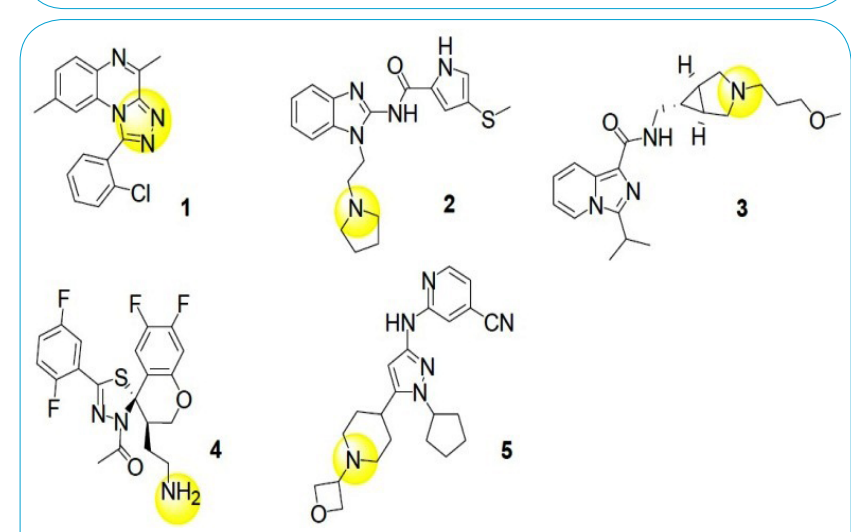

Figure 2: The structures of recently reported brain-penetrant compounds.

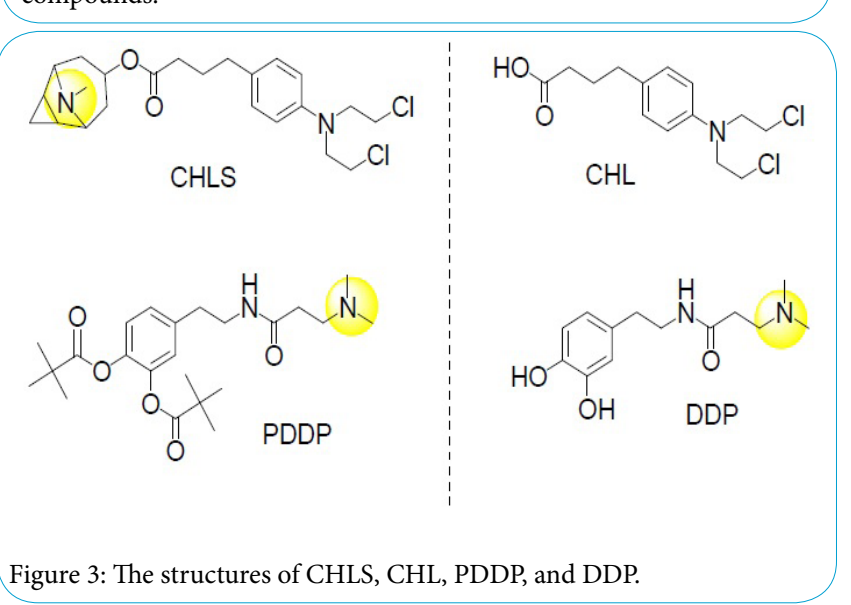

and OCTN2 substrate) and reserpine (vesicular monoamine transporter inhibitor). These results implied that CHLS was transported by the unidentified $\mathrm{H}^{+} / \mathrm{OC}$ antiporter [16]. Moreover, $\mathrm{N}$-3,4-bis(pivaloyloxy)domapine-3-(dimethylamino)propanamide (PDDP), the conjugate of 3,4-bis(pivaloyloxy)dopamine and $N, N$ dimethylamino group through the linker chain, was transported into brain across the $\mathrm{BBB}$ in in vivo assay based on intravenous injection 
Citation: Tashima T (2016) Intelligent Design of Brain-penetrant Compounds Based on Transporter-Conscious Drug Design. Int J Clin Pharmacol Pharmacother 1: 118. doi: http://dx.doi.org/10.15344/2016/ijccp/118

Page 3 of 3

in rats, which exhibited about 6.90 -fold greater absorption into brain compared to 3,4-bis(pivaloyloxy)dopamine (BPD). The transportation of PDDP (Figure 3) was inhibited by pyrilamine, propranolol, and imipramine and was not inhibited by choline (substrate of choline transport system), L-carnitine (OCTN2 substrate), and TEA (OCT13 , OCTN1, and OCTN2 substrate) in in vitro assay using bEnd. 3 cells. These results implied that PDDP was transported by the unidentified $\mathrm{H}^{+} / \mathrm{OC}$ antiporter. Intriguingly, $\mathrm{N}$-(dopamine)-3-(dimethylamino) propanamide (DDP), which was the corresponding unprotected PDDP, was not the substrate of the unidentified $\mathrm{H}^{+} / \mathrm{OC}$ antiporter [17]. Thus, it was suggested that the hydrophobicity of substrates was very important to the transportation based on of the unidentified $\mathrm{H}^{+} /$ $\mathrm{OC}$ antiporter, in addition to the existence of $\mathrm{N}$-containing groups as transporter recognition unit.

\section{Conclusions}

Many drugs have been dropped out in preclinical tests and clinical trials due to poor performance of absorption, distribution, excretion, and toxicity (ADMET). Particularly, the cell permeability is a serious problem in drug discovery and development. Moreover, as for CNS drug, the permeation into brain across the BBB is a crucial aspect. However, the carrier-mediated transport system using SLC transporters can solve these permeable problems. At the BBB, in small intestine, and in cancer cells, MDR1 is expressed in the apical (luminal) membrane and excludes drugs passing through the cell membrane based on passive diffusion, from the membrane to the outside of cells. Thus, if only such drugs are transported by coexpressed SLC transporters, these drugs can be delivered into brain across the BBB (Figure 1). Therefore, transporter-conscious drug design is very important in ADMET. Furthermore, it is well-known that compounds possessing $\mathrm{N}$-containing groups are delivered into brain across the BBB. Aliphatic amines were generally ammonium ions under physiological $\mathrm{pH}$ condition and thus were not able to penetrate the cell membrane by passive diffusion. Accordingly, it was suggested that compounds possessing $\mathrm{N}$-containing groups were interacted with transporters. In fact, the proton-coupled organic cation $\left(\mathrm{H}^{+} / \mathrm{OC}\right)$ antiporter, whose characterization such as amino acid sequence and topology remains unclear, is considered as a key transporter at the BBB among transporters which transported compounds possessing $\mathrm{N}$-containing groups. In drug design, $\mathrm{N}$-containing groups as the structural common features of arbitrary amino transporter substrates should be incorporated into novel designed compounds as transporter recognition unit. The permeability of such designed compounds should be evaluated in in vitro assay using appropriate cells. Subsequently, the evaluated compounds should be structurally redesigned based on the permeation results and be reevaluated. Currently, I think that synthetic chemists or medicinal chemists may penetrate transporter-consciously designed drugs into brain across the wall between blood and brain like the lead character in the novel or musical of "Le Passe-muraille" and exhibit their prowess at the stages of clinical trials.

\section{Abbreviations}

CNS: Central Nervous System; BBB: Blood Brain Barrier; MDR1: Multidrug Resistance 1; SLC transporter: Solute Carrier Transporter; ABC-transporter: ATP Binding Cassette Transporter; OC: Organic Cation

\section{Competing Interests}

The author declare that they have no competing interests.

\section{Acknowledgments}

This short review is just my opinion based on or inferred from available published articles and public knowledge. Thus, the intellectual property rights are not infringed upon.

\section{References}

1. Stenehjem DD, Hartz AM, Bauer B, Anderson GW (2009) Novel and emerging strategies in drug delivery for overcoming the blood-brain barrier, Future Med Chem 1: 1623-1641.

2. Chen Y, Liu L (2012) Modern methods for delivery of drugs across the blood-brain barrier. Advanced Drug Delivery Reviews 64: 640-665.

3. International Transporter Consortium, Giacomini KM, Huang SM, Tweedie DJ, Benet LZ, Brouwer KL, et al. (2010) Membrane transporters in drug development. Nat Rev Drug Disc 9: 215-236.

4. Kimura Y, Morita S, Matsuo M, Ueda K (2007) Mechanism of multidrug recognition by MDR1/ABCB1. Cancer Sci 98: 1303-1308.

5. Tashima $T$ (2015) Intriguing possibilities and beneficial aspects of transporter-conscious drug design. Bioorg Med Chem 23: 4119-4131.

6. Nakanishi T, Tamai I (2011) Solute carrier transporters as targets for drug delivery and pharmacological intervention for chemotherapy. J Pharm Sci 100: 3731.

7. Mehta DC, Short JL, Nicolazzo JA (2013) Memantine transport across the mouse blood-brain barrier is mediated by a cationic influx $\mathrm{H}^{+}$antiporter. $\mathrm{Mol}$ Pharm 10: 4491-4498.

8. André P, Debray M, Scherrmann JM, Cisternino S (2009) Clonidine transport at the mouse blood-brain barrier by a new $\mathrm{H}^{+}$antiporter that interacts with addictive drugs. J Cereb Blood Flow Metab 29: 1293-1304.9.

9. Shimomura K, Okura T, Kato S, Couraud PO, Schermann JM, et al. (2013) Functional expression of a proton-coupled organic cation $\left(\mathrm{H}^{+} / \mathrm{OC}\right)$ antiporter in human brain capillary endothelial cell line hCMEC/D3, a human bloodbrain barrier model. Fluids Barriers CNS 10: 8 .

10. Redrobe JP, Rasmussen LK, Christoffersen CT, Bundgaard C, Jørgensen M (2015) Characterisation of Lu AF33241: A novel, brain-penetrant, dual inhibitor of phosphodiesterase (PDE) 2A and PDE10A.Eur J Pharm 761: 79-85.

11. Sasmal PK, Krishna C V, Adabala S, Roshaiah SS, Rawoof M, et al (2015) Optimisation of in silico derived 2-aminobenzimidazole hits as unprecedented selective kappa opioid receptor agonists. Bioorg Med Chem Lett 25: 887-892.

12. Nirogi R, Mohammed AR, Shinde AK, Bogaraju N Gagginapalli SR, et al. (2015) Discovery of novel spiro 1,3,4-thiadiazolines as potent, orally bioavailable and brain penetrant KSP inhibitors. Eur J Med Chem 103: 289-301.

13. Mansoor UF, Angeles AR, Dai C, Yang L, Vitharana D, et al. (2015) Discovery of novel spiro 1,3,4-thiadiazolines as potent, orally bioavailable and brain penetrant KSP inhibitors. Bioorg Med Chem 23: 2424-2434.

14. Patel S, Harris SF, Gibbons P, Deshmukh G, Gustafson A, et al. (2015) Scaffold-Hopping and Structure-Based Discovery of Potent, Selective, And Brain Penetrant N-(1H-Pyrazol-3-yl)pyridin-2-amine Inhibitors of Dual Leucine Zipper Kinase (DLK, MAP3K12). J Med Chem 58: 8182-8199.

15. Ariza M, Kolb HC, Moechars D, Rombouts F, Andrés Jl (2015) J Med Chem 58: $4365-4382$

16. Wang X, Li J, Xu C, Li Y, Gong T, et al. (2014) Scopine as a Novel BrainTargeting Moiety Enhances the Brain Uptake of Chlorambucil. Bioconjugate Chem 25: 2046-2054

17. Li Y, Zhou Y, Qi B, Gong T, Sun X, et al. (2014) Brain-specific delivery of dopamine mediated by $n, n$-dimethyl amino group for the treatment of Parkinson's disease. Mol Pharm 11: 3174-3185. 